\title{
Pediatric sepsis biomarkers for prognostic and predictive enrichment
}

\author{
Hector R. Wong ${ }^{1,2 \bowtie}$ \\ (c) The Author(s), under exclusive licence to the International Pediatric Research Foundation, Inc 2021
}

Sepsis is a major public health problem in children throughout the world. Given that the treatment guidelines emphasize early recognition, there is interest in developing biomarkers of sepsis, and most attention is focused on diagnostic biomarkers. While there is a need for ongoing discovery and development of diagnostic biomarkers for sepsis, this review will focus on less wellknown applications of sepsis biomarkers. Among patients with sepsis, the biomarkers can give information regarding the risk of poor outcome from sepsis, risk of sepsis-related organ dysfunction, and subgroups of patients with sepsis who share underlying biological features potentially amenable to targeted therapeutics. These types of biomarkers, beyond the traditional concept of diagnosis, address the important concepts of prognostic and predictive enrichment, which are key components of bringing the promise of precision medicine to the bedside of children with sepsis.

Pediatric Research (2022) 91:283-288; https://doi.org/10.1038/s41390-021-01620-5

\section{INTRODUCTION}

Current treatment guidelines for sepsis emphasize a high index of suspicion for early detection of sepsis and timely administration of antibiotics as key principles for improving outcomes ${ }^{1}$. Consequently, there is considerable interest in sepsis biomarkers, which provide information regarding the treatment guideline application. When considering "sepsis biomarkers" it is important to understand the clinical context and what information one seeks to derive from the biomarker data.

Evaluating febrile children, who are otherwise clinically undifferentiated, is a common challenge for pediatricians. In this context, the biomarker data can be useful in differentiating those with infection from those with a sterile source of systemic inflammation as the underlying cause of fever. Further, the biomarkers can also be useful in differentiating those with bacterial infection from those with a viral infection, as this would help in deciding whether or not to prescribe antibiotics. Procalcitonin, although imperfect, has emerged as a widely used biomarker to address these challenges in both the ambulatory and inpatient settings ${ }^{2}$. There are also emerging gene expression panels that show substantial promise in addressing these clinical challenges ${ }^{3-12}$. These panels have the potential to capture the complexity of the human response to infection more accurately than single biomarker strategies, and therefore can have added value when compared with single biomarker strategies ${ }^{13}$.

While diagnostic biomarkers for sepsis are of great clinical importance, this important topic will not be the focus of this review, as it is well covered in the recent review literature. Rather, this review will address other important clinical questions, less well covered in the existing literature, that could be addressable by the discovery and development of reliable biomarkers. The clinical questions addressed in this review refer to patients diagnosed with sepsis based on the current criteria ${ }^{14}$ and include: Among patients with sepsis, what is the risk for a poor outcome? Among patients with sepsis, what is the risk for clinically important organ failure? Among patients with sepsis, do there exist identifiable subgroups that share common underlying biological features?

As will be discussed in subsequent sections, effectively addressing these questions, via the discovery and development of biomarkers, is fundamental to bringing the promise of precision medicine to the bedside of children with sepsis ${ }^{15}$.

\section{BIOMARKERS TO ESTIMATE THE RISK OF POOR OUTCOME FROM SEPSIS}

Mortality secondary to sepsis remains a major public health problem for children in the United States and around the world ${ }^{16-18}$. Reliably estimating baseline mortality risk from pediatric sepsis is a form of prognostic enrichment, which refers to the selection of a patient cohort more likely to have an outcome of interest when compared with an unselected cohort $^{15,19}$. Prognostic enrichment is fundamental to clinical decision making, resource allocation, quality improvement efforts, and research. While many biomarkers have been evaluated to estimate the risk for a poor outcome from sepsis, few have been validated in a sufficiently rigorous manner to warrant clinical adoption as a prognostic enrichment strategy.

The Pediatric Sepsis Biomarker Risk Model (PERSEVERE) incorporates a panel of serum protein biomarkers, measured within $24 \mathrm{~h}$ of a sepsis diagnosis, to estimate baseline risk of mortality among critically ill children with septic shock ${ }^{20-23}$. Figure 1 depicts the general approach for identifying the PERSEVERE biomarkers by leveraging the discovery potential of transcriptomics based on whole-blood-derived RNA. In a cohort of

${ }^{1}$ Cincinnati Children's Hospital Medical Center and Cincinnati Children's Research Foundation, Cincinnati, OH, USA. ${ }^{2}$ Department of Pediatrics, University of Cincinnati College of Medicine, Cincinnati, OH, USA. ${ }^{凶}$ email: hector.wong@cchmc.org 


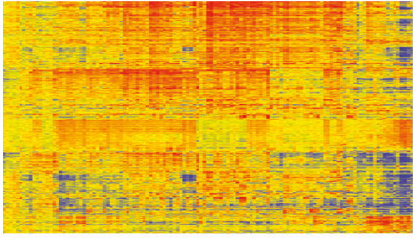

Generation of transcriptomic data using whole-blood-derived RNA

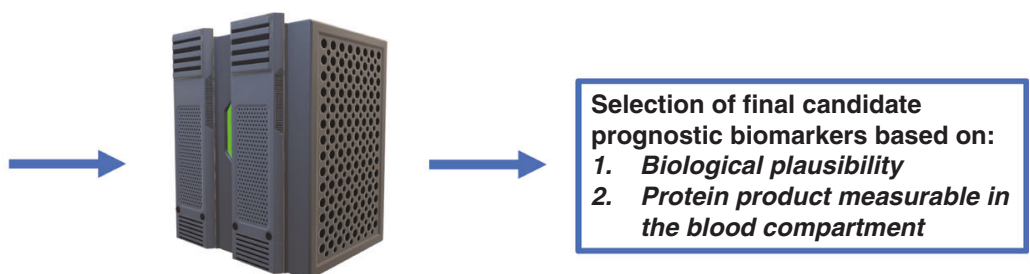

Bioinformatics to identify gene expression associated with 28-day mortality

Fig. 1 General workflow for leveraging transcriptomic data for the discovery of candidate prognostic (PERSEVERE) biomarkers for pediatric septic shock. See text for details.

Table 1. Candidate PERSEVERE biomarkers.

\begin{tabular}{ll} 
Gene symbol & Description \\
\hline CCL3 & C-C chemokine ligand 3 \\
\hline CCL4 & C-C chemokine ligand 4 \\
\hline ELA2 & Neutrophil elastase 2 \\
\hline GZMB & Granzyme B \\
\hline HSPA1B & Heat-shock protein 70 kDa 1B \\
\hline IL1A & Interleukin-1 $\alpha$ \\
\hline IL8 & Interleukin-8 \\
\hline LCN2 & Lipocalin-2 \\
\hline LTF & Lactotransferrin \\
\hline MMP8 & Metallopeptidase-8 \\
\hline RETN & Resistin \\
\hline THBS1 & Thrombospondin-1 \\
\hline
\end{tabular}

critically ill children with septic shock, complementary bioinformatics and machine learning approaches were employed to derive a list of candidate predictor genes for 28-day mortality ${ }^{20,24}$. From over 100 candidate genes, the list was further defined using two a priori criteria: (1) biological plausibility linking the candidate predictor gene and sepsis-related pathobiology and (2) the ability to measure the gene product (i.e., protein) in the blood compartment. Based on these criteria, 12 candidate biomarkers were selected for further evaluation (Table 1).

The serum protein concentrations of these 12 biomarkers were then measured in a cohort of children with septic shock to model the risk of 28-day mortality using Classification and Regression Tree methodology ${ }^{20}$. The resulting model, PERSEVERE, included five of the original 12 candidate biomarkers (CCL3, IL8, HSPA1B, GZMB, and MMP8) and age as predictor variables. Subsequently, PERSEVERE was recalibrated to yield PERSEVERE II, which includes admission platelet count as an additional predictor variable ${ }^{25}$. The PERSEVERE II decision rules were locked and prospectively applied to a validation cohort of children with septic shock, yielding an area under the receiver operating (AUROC) of 0.86 (95\% confidence interval (CI) $0.81-0.90)$, a sensitivity of $90 \%(95 \% \mathrm{Cl} 78-96)$, and a specificity of $69 \%(95 \% \quad \mathrm{Cl} 64-73)$ for distinguishing non-survivors from survivors at 28 days $^{26}$. The PERSEVERE II decision tree is shown in Fig. 2.

As a test of internal validity, the true-negative patients (those correctly predicted to survive) identified by PERSEVERE II were compared with the false-positive patients (those incorrectly predicted to die by 28 days). False-positive patients had greater derangements of physiological variables and greater organ failure burden when compared with the true-negative patients ${ }^{26}$. This suggests that the false-positive subjects identified by PERSEVERE II were indeed at higher risk of mortality, but that risk was perhaps mitigated by clinical interventions. Related to this observation, in a preliminary investigation, the PERSEVERE biomarkers appeared to have utility for estimating the risk of poor functional and quality of life outcomes among children who survived the acute stage of sepsis and were discharged from the hospital ${ }^{27}$. Collectively, the accumulating evidence, including successful prospective validation in a highly heterogeneous cohort of patients, suggests that PERSEVERE II could have clinical utility as a prognostic enrichment tool. Interestingly, the PERSEVERE biomarkers also have utility for estimating the risk of mortality in mice subjected to experimental sepsis $^{26}$. This suggests that the biological pathways to poor outcome from sepsis are conserved in experimental models, thus providing an opportunity to dissect the mechanistic and biological links between the PERSEVERE biomarkers and the pathways to poor outcome from sepsis.

Another recent endeavor to develop prognostic enrichment tools for sepsis involved a collaboration among three independent research groups ${ }^{28}$. Here, the investigators cataloged all publicly available transcriptomic data involving adults, children, and neonates with sepsis and set out to develop independent mortality prediction models. Using independent, data-driven, machine learning approaches, each investigative group developed mortality prediction models using the same input data and all models performed well upon validation. Of note, the models developed by these three independent groups yielded a combined total of 65 candidate predictor genes ${ }^{28}$. Among these, 11 overlapped with the genes identified in the initial discovery phase of the PERSEVERE biomarkers (Table 2), thus adding a measure of internal validity among the various models. A recent approach employing artificial intelligence and transcriptomic data further confirmed the reliability of these models with respect to the identified classifier genes ${ }^{29}$.

\section{Biomarkers to estimate the risk of sepsis-associated organ dysfunction}

While all organs are at risk of dysfunction during sepsis, acute kidney injury (AKI) is a particularly common complication of sepsis, and independently contributes to increased sepsis-related morbidity and mortality ${ }^{30,31}$. The underlying pathology of sepsisassociated AKI involves a complex and incompletely understood interplay of deranged perfusion, oxidant stress, and inflammation. Consequently, there is interest in developing strategies to identify children at greater risk of developing sepsis-associated $\mathrm{AKI}^{31}$. This form of prognostic enrichment focused on the development of sepsis-associated AKI, rather than mortality, could enable the targeted application of renal protective strategies and renal replacement therapy, and in the future could serve to inform enrollment for interventional clinical trials to mitigate sepsisassociated AKI.

Recently, the PERSEVERE biomarkers were used to develop a model to estimate the risk of sepsis-associated AKI in a cohort of critically ill children with septic shock ${ }^{32}$. The primary outcome variable for this study was the development of severe AKI on day 3 of septic shock. Severe sepsis-associated AKI was defined as Kidney Disease Improving Global Outcomes stage 2 AKI or 


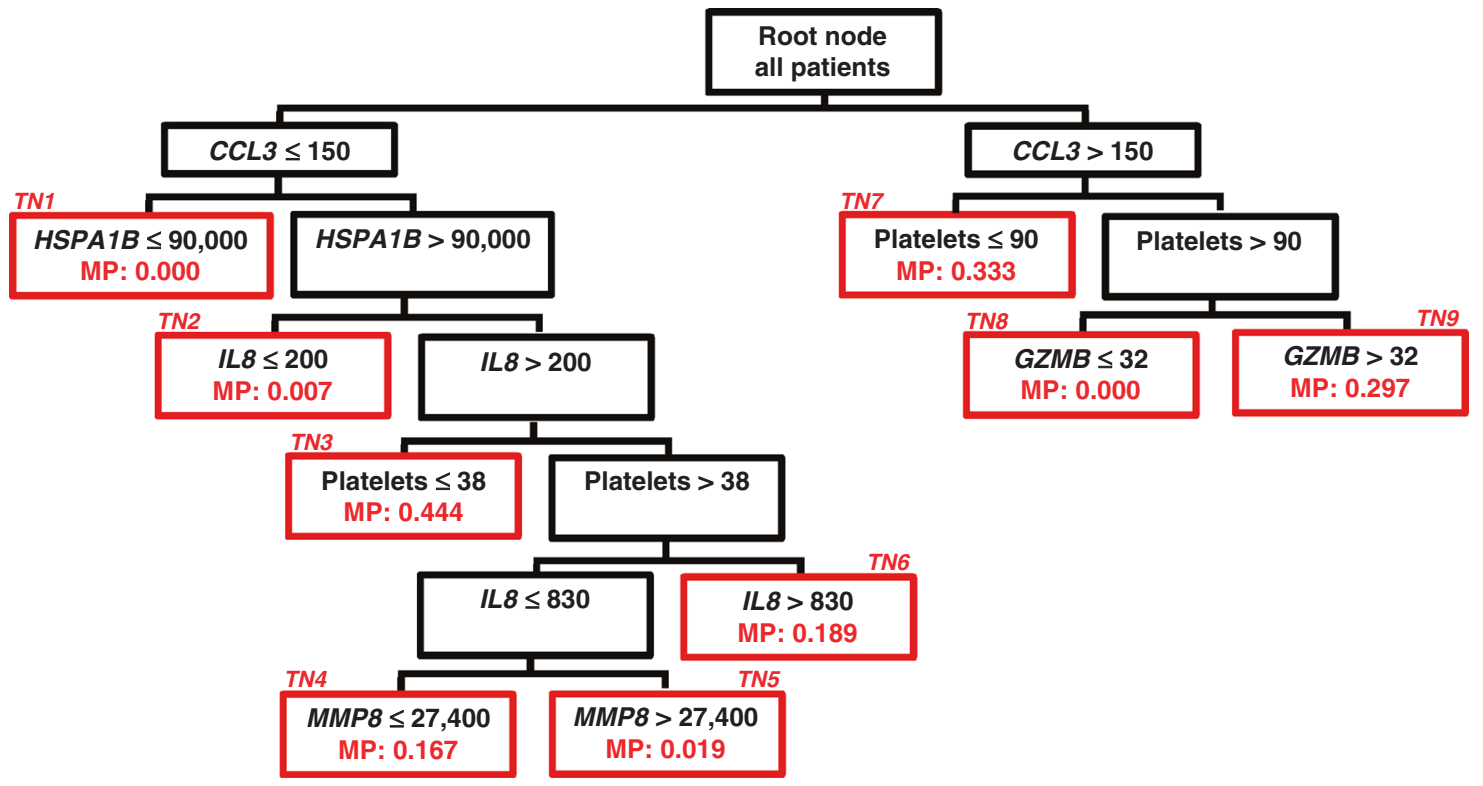

Fig. 2 The PERSEVERE II decision tree. All patients begin at the root node and are subsequently partitioned to daughter nodes using biomarker-based criteria as indicated at the top of each node. All biomarker data are shown as $\mathrm{pg} / \mathrm{ml}$, and platelet data are shown as the number of platelets $(\mathrm{K} / \mu \mathrm{l})$. Daughter nodes that can no longer be partitioned are called terminal nodes (TNs) and shown in red outline. The terminal nodes are used to assign a baseline mortality probability to a patient classified to a given terminal node. The baseline mortality probability (MP) corresponding to each terminal node is indicated in red font within each terminal node and is derived from the published PERSEVERE II model. These baseline mortality risks are used for the construction of the AUROC. For calculation of the diagnostic test characteristics, the mortality probability is dichotomized into those who are predicted to survive and those who are predicted to not survive by 28 days. Patients allocated to TN1, TN2, TN5, and TN8 (mortality risk $0.000-0.019$ ) are classified as predicted survivors. Patients allocated to TN3, TN4, TN6, TN7, and TN9 are classified as predicted non-survivors (mortality risk $0.167-0.444$ ).

Table 2. Genes common to a community approach for identification of prognostic enrichment genes and the original working gene list for the PERSEVERE biomarkers.

\begin{tabular}{ll}
\hline Gene symbol & Description \\
\hline CD24 & CD24 molecule \\
\hline CEACAM8 & $\begin{array}{l}\text { Carcinoembryonic antigen-related cell adhesion } \\
\text { molecule } 8\end{array}$ \\
\hline CKS2 & CDC28 protein kinase regulatory subunit 2 \\
\hline CX3CR1 & C-X3-C motif chemokine receptor 1 \\
\hline DDIT4 & DNA damage-inducible transcript 4 \\
\hline EMR3 & Adhesion G-protein-coupled receptor E3 \\
\hline GOS2 & G0/G1 switch 2 \\
\hline IL8 & Interleukin-8, C-X-C motif chemokine ligand 8 \\
\hline MAFF & MAF bZIP transcription factor F \\
\hline RGS1 & Regulator of G-protein signaling 1 \\
\hline TGFBI & Transforming growth factor beta induced \\
\hline
\end{tabular}

greater, which is at least a two-fold increase in serum creatinine concentration compared to baseline.

The derived model estimated the risk of severe sepsisassociated AKI with an AUROC of 0.95 (95\% Cl $0.92-0.98)$, a sensitivity of $92 \%(95 \% \mathrm{Cl} 82-97)$, and a specificity of $89 \%(95 \% \mathrm{Cl}$ $85-92)^{32}$. A ten-fold cross-validation procedure yielded a summary AUROC of 0.88 , and when tested using a historical cohort, the model had an AUROC of 0.83 (95\% Cl $0.79-0.88)$. In addition, the model was able to identify patients with evidence of AKI at the time of admission to the pediatric intensive care unit, but who would go on to recover renal function by day 3 of septic shock. This model requires prospective validation, but its performance characteristics warrant further exploration as a potential prognostic enrichment strategy for sepsis-associated AKI.
In a conceptually related study, the PERSEVERE biomarkers were evaluated for their ability to identify the risk of clinical deterioration among immunocompromised, hospitalized patients being evaluated for possible infection ${ }^{33}$. The enrollment criteria captured patients in whom a blood culture was obtained as part of the workup for suspected infection. The PERSEVERE biomarkers were measured at the same time as the blood culture sample. Clinical deterioration was defined by a set of criteria to codify evolving organ failure requiring intensive care and was tracked up to $72 \mathrm{~h}$ from enrollment. This study showed that the PERSEVERE biomarkers were able to identify patients more likely to experience clinical deterioration (i.e., evolving organ dysfunction) with a sensitivity of $86 \%$ and a negative predictive value of $97 \%$. This was a single-center study that requires validation, as well as expansion to patients being evaluated for infection who are not immunocompromised. For example, it could be productive to evaluate the PERSEVERE biomarkers among immune-competent children who are being evaluated in the ambulatory setting for possible infection. In this context, the PERSEVERE biomarkers could be evaluated for their ability to identify patients with a greater likelihood of clinical deterioration, therefore informing treatment and disposition decisions.

\section{Gene expression-based endotypes of septic shock}

Endotypes represent subgroups of heterogeneous clinical syndromes as defined by commonalities of underlying biology ${ }^{19}$. Recently, there has been considerable interest in identifying endotypes of septic shock using genome-wide expression profiling $^{34}$. The underlying premise of these efforts is that unbiased transcriptomic studies can reveal endotypes of sepsis based on coordinated gene expression patterns (i.e., biomarkers) shared among endotypes. The general approach involves unsupervised clustering algorithms based on commonalities of gene expression, agnostic to patient characteristics, clinical trajectory, and outcome. Ultimately, the goal is to leverage gene 
expression-based endotyping as a predictive enrichment tool. Distinct from prognostic enrichment, predictive enrichment refers to the selection of a patient cohort in whom an intervention is more likely to be effective based on underlying biology when compared with an unselected cohort ${ }^{15}$. Like prognostic enrichment, predictive enrichment is thought to be a key enabler of precision medicine.

In 2016, Davenport and colleagues reported two gene expressionbased endotypes of sepsis among adults, termed sepsis response signature 1 and 2 (SRS1 and SRS2) ${ }^{35}$. The gene expression profile defining SRS1 corresponded to endotoxin tolerance, repression of human leukocyte antigen class II, and T cell exhaustion. Patients assigned to SRS1 had a higher mortality rate compared with patients assigned to SRS2. The existence of SRS1 and SRS2 was validated in a subsequent study of adults with sepsis and appears to be applicable among patients who have either a pulmonary or intra-abdominal source of sepsis ${ }^{36}$.

In 2017, Scicluna et al. identified four gene expression-based endotypes among adults with sepsis, termed Mars1 to $4^{37}$. Patients assigned to the Mars1 endotype had the highest risk of mortality, and like the SRS1 endotype, the Mars1 endotype was characterized by repression of gene programs corresponding to innate and adaptive immunity.

In 2018, Sweeney et al. leveraged virtually all publicly available transcriptomic data involving adults, children, and neonates with sepsis to identify three endotypes of sepsis ${ }^{38,39}$. These were termed inflammopathic, adaptive, and coagulopathic, reflecting their respective endotype-defining gene expression signatures. The coagulopathic endotype was associated with the highest mortality rate among the three endotypes. Recently, the existence of these three endotypes and their association with mortality was reported among critically ill patients with coronavirus disease $2019^{40}$.

Analogous gene expression endotypes of septic shock were reported among critically ill children in 2009, and subsequently validated $^{41-44}$. Pediatric septic shock endotypes $A$ and $B$ are defined by 100 genes corresponding to the adaptive immune system and glucocorticoid receptor signaling. Endotype A assignment is independently associated with higher mortality and organ failure burden when compared to endotype B assignment. Approximately one-third of children with septic shock change endotype assignment during the initial 3 days of septic shock ${ }^{45}$. Children who persist as endotype A during the entire 3-day acute period of septic shock are at the highest risk for poor outcome. Finally, an interaction between younger age, assignment to endotype $A$, and increased risk of mortality was reported among adults with septic shock ${ }^{46}$.

The aforementioned studies share an approach to endotype identification based on transcriptomic data generated using whole-blood-derived RNA and clustering algorithms to classify patients with sepsis into subgroups that share common patterns of gene expression. All the studies report associations between endotype assignment and mortality, but this is not the primary intent of identifying endotypes. Rather, the primary intent of endotype identification among patients with sepsis is to identify subgroups with common underlying biological features that could potentially be amendable to targeted therapies. This embodies the concepts of predictive enrichment and precision medicine. For example, all the endotyping strategies ostensibly identify a subgroup in whom dysregulation of the adaptive immune system is inferred based on the endotypedefining gene expression profile. If these endotyping strategies are truly identifying subgroups of patients with alterations of the adaptive immune system, it could have major implications for designing trials targeting modulation of the adaptive immune system among patients with sepsis ${ }^{47,48}$. On a broader level, these endotyping strategies have the potential to manage the challenge of heterogeneity of treatment effect typically observed in interventional trials involving patients with septic shock.

Heterogeneity of treatment effect among patients with sepsis is perhaps best exemplified when considering the role of adjunctive corticosteroids for patients with septic shock, which has been intensely debated since the $1960 \mathrm{~s}^{49}$. Adjunctive corticosteroids are thought to have a potential therapeutic role in septic shock given their pluripotent effects on the cardiovascular system, inflammation, and immunity. Across multiple, large, randomized trials, adjunctive corticosteroids consistently reduce vasopressor requirements, but there has not been a consistently positive effect on patient survival ${ }^{50-53}$. Recently, it has been suggested that gene expression-based endotyping could provide a means to better understand and manage the heterogeneity of treatment effect for adjunctive corticosteroids among patients with septic shock ${ }^{54}$.

The Vasopressin vs. Norepinephrine as Initial Therapy in Septic Shock (VANISH) trial randomized adults with septic shock to hydrocortisone or placebo upon reaching a predefined vasopressor dose ${ }^{55}$. A subset of the VANISH cohort also had corresponding transcriptomic data, and Antcliffe et al. used these data to assign the cohort to the aforementioned SRS1 or SRS2 endotypes ${ }^{56}$. They found that hydrocortisone use was independently associated with increased odds of mortality among patients assigned to the SRS2 endotype.

Among children with septic shock, observational data have consistently demonstrated that corticosteroid prescription is associated with increased odds of mortality among patients assigned to pediatric septic shock endotype $A^{43,45}$. This observation remains after adjusting for baseline illness severity, age, and comorbidity burden. Recently, the publicly available transcriptomic data from the VANISH trial was used to cross-assign adults with septic shock to pediatric septic shock endotypes $A$ or $B^{57}$. This study corroborated the association between corticosteroids and increased mortality risk among patients assigned to endotype A.

Another line of investigation used observational data to determine if there is a subgroup of children with septic shock that is more likely to benefit from adjunctive corticosteroids ${ }^{58}$. This study combined PERSEVERE-based mortality risk stratification with endotyping and reported that among endotype $B$ patients with an intermediate to the high baseline risk of mortality, adjunctive corticosteroids were associated with a more than ten-fold reduction of risk for poor outcome. This study, albeit based on observational data, illustrates the concept of how prognostic enrichment (PERSEVERE) and predictive enrichment (endotyping) can potentially be combined to identify a subset of patients more likely to have a beneficial response to adjunctive corticosteroids. The concept is currently being tested prospectively among adults (NCT04280497) and children (NCT03401398) with septic shock. A major next step for implementing these strategies at the bedside is the development of rapid assay platforms that can generate clinically actionable information in a clinically relevant timeframe.

\section{CONCLUSION}

Leveraging biomarkers to identify which patients have sepsis continues to be an important and well-recognized area of study. Equally important, but less well recognized, is the discovery and development of biomarkers for prognostic and predictive enrichment among patients with sepsis. This area has progressed considerably over the last few years in the pediatric domain. While a great deal of validation and development work remains, biomarkers for prognostic and predictive enrichment hold the promise of bringing precision medicine to the bedside of children with sepsis. This would include the enhancement of clinical trial design, the development of more targeted therapies, and bringing biological precision to quality improvement efforts. 


\section{REFERENCES}

1. Weiss, S. L. et al. Surviving sepsis campaign international guidelines for the management of septic shock and sepsis-associated organ dysfunction in children. Pediatr. Crit. Care Med. 21, e52-e106 (2020).

2. Leticia Fernandez-Carballo, B., Escadafal, C., MacLean, E., Kapasi, A. J. \& Dittrich, S. Distinguishing bacterial versus non-bacterial causes of febrile illness - a systematic review of host biomarkers. J. Infect. 82, 1-10 (2021).

3. Sweeney, T. E., Shidham, A., Wong, H. R. \& Khatri, P. A comprehensive timecourse-based multicohort analysis of sepsis and sterile inflammation reveals a robust diagnostic gene set. Sci. Transl. Med. 7, 287 ra271 (2015).

4. Sweeney, T. E. \& Khatri, P. Comprehensive validation of the FAIM3:PLAC8 ratio in time-matched public gene expression data. Am. J. Respir. Crit. Care Med. 192, 1260-1261 (2015).

5. Andres-Terre, M. et al. Integrated, multi-cohort analysis identifies conserved transcriptional signatures across multiple respiratory viruses. Immunity $\mathbf{4 3}$, 1199-1211 (2015).

6. Sweeney, T. E., Wong, H. R. \& Khatri, P. Robust classification of bacterial and viral infections via integrated host gene expression diagnostics. Sci. Transl. Med. 8, 346ra391 (2016).

7. Mayhew, M. B. et al. A generalizable 29-mRNA neural-network classifier for acute bacterial and viral infections. Nat. Commun. 11, 1177 (2020).

8. Ducharme, J. et al. A multi-mRNA host-response molecular blood test for the diagnosis and prognosis of acute infections and sepsis: proceedings from a clinical advisory panel. J. Pers. Med. 10, 266 (2020).

9. Thair S. et al. Gene expression-based diagnosis of infections in critically ill patients-prospective validation of the sepsis metascore in a longitudinal severe trauma cohort. Crit. Care Med. https://doi.org/10.1097/CCM.0000000000005027, Online ahead of print (2021).

10. Mahajan, P. et al. Association of RNA biosignatures with bacterial infections in febrile infants aged 60 days or younger. JAMA 316, 846-857 (2016).

11. Herberg, J. A. et al. Diagnostic test accuracy of a 2-transcript host RNA signature for discriminating bacterial vs viral infection in febrile children. JAMA 316, 835-845 (2016)

12. Sampson, D. et al. Blood transcriptomic discrimination of bacterial and viral infections in the emergency department: a multi-cohort observational validation study. BMC Med. 18, 185 (2020).

13. Conway, S. R. \& Wong, H. R. Biomarker panels in critical care. Crit. Care Clin. 36 , 89-104 (2020).

14. Goldstein, B., Giroir, B. \& Randolph, A. International pediatric sepsis consensus conference: definitions for sepsis and organ dysfunction in pediatrics. Pediatr. Crit. Care Med. 6, 2-8 (2005).

15. Stanski, N. L. \& Wong, H. R. Prognostic and predictive enrichment in sepsis. Nat. Rev. Nephrol. 16, 20-31 (2020).

16. Weiss, S. L. et al. Global epidemiology of pediatric severe sepsis: the sepsis prevalence, outcomes, and therapies study. Am. J. Respir. Crit. Care Med. 191, 1147 (2015).

17. Fleischmann, C. et al. Global incidence and mortality of neonatal sepsis: a systematic review and meta-analysis. Arch. Dis. Child. https://doi.org/10.1136/archdischild-2020320217, Online ahead of print (2021)

18. Rudd, K. E. et al. Global, regional, and national sepsis incidence and mortality, 1990-2017: analysis for the Global Burden of Disease Study. Lancet 395, 200-211 (2020).

19. Prescott, H. C., Calfee, C. S., Thompson, B. T., Angus, D. C. \& Liu, V. X. Toward smarter lumping and smarter splitting: rethinking strategies for sepsis and acute respiratory distress syndrome clinical trial design. Am. J. Respir. Crit. Care Med. 194, 147-155 (2016).

20. Wong, H. R. et al. The pediatric sepsis biomarker risk model. Crit. Care 16, R174 (2012).

21. Wong, H. R. et al. Testing the prognostic accuracy of the updated pediatric sepsis biomarker risk model. PLOS ONE 9, e86242 (2014).

22. Wong, H. R. et al. Improved risk stratification in pediatric septic shock using both protein and mRNA biomarkers. PERSEVERE-XP. Am. J. Respir. Crit. Care Med. 196, 494-501 (2017).

23. Wong, H. R. et al. Interleukin-8 as a stratification tool for interventional trials involving pediatric septic shock. Am. J. Respir. Crit. Care Med. 178, 276-282 (2008).

24. Kaplan, J. M. \& Wong, H. R. Biomarker discovery and development in pediatric critical care medicine. Pediatr. Crit. Care Med. 12, 165-173 (2011).

25. Wong, H. R. et al. Pediatric sepsis biomarker risk Model-Il: redefining the pediatric sepsis biomarker risk model with septic shock phenotype. Crit. Care Med. 44, 2010-2017 (2016).

26. Wong, H. R. et al. Prospective clinical testing and experimental validation of the Pediatric Sepsis Biomarker Risk Model. Sci. Transl. Med. 11, eaax9000 (2019).

27. Wong, H. R. et al. Biomarkers for estimating risk of hospital mortality and longterm quality-of-life morbidity after surviving pediatric septic shock: a secondary analysis of the life after pediatric sepsis evaluation investigation. Pediatr. Crit. Care Med. 22, 8-15 (2021).

28. Sweeney, T. E. et al. A community approach to mortality prediction in sepsis via gene expression analysis. Nat. Commun. 9, 694 (2018).

29. Banerjee, S., Mohammed, A., Wong, H. R., Palaniyar, N. \& Kamaleswaran, R. Machine learning identifies complicated sepsis course and subsequent mortality based on 20 genes in peripheral blood immune cells at $24 \mathrm{~h}$ post-ICU admission. Front. Immunol. 12, 592303 (2021).

30. Manrique-Caballero, C. L., Del Rio-Pertuz, G. \& Gomez, H. Sepsis-associated acute kidney injury. Crit. Care Clin. 37, 279-301 (2021).

31. Stanski, N. L., Cvijanovich, N. Z., Fitzgerald, J. C., Bigham, M. T. \& Wong, H. R. Severe acute kidney injury is independently associated with mortality in children with septic shock. Intens. Care Med. 46, 1050-1051 (2020).

32. Stanski, N. L. et al. PERSEVERE biomarkers predict severe acute kidney injury and renal recovery in pediatric septic shock. Am. J. Respir. Crit. Care Med. 201, 848-855 (2020).

33. Jacobs, L. et al. The Pediatric Sepsis Biomarker Risk Model (PERSEVERE) biomarkers predict clinical deterioration and mortality in immunocompromised children evaluated for infection. Sci. Rep. 9, 424 (2019).

34. DeMerle, K. M. et al. Sepsis subclasses: a framework for development and interpretation. Crit. Care Med. 49, 748-759 (2021).

35. Davenport, E. E. et al. Genomic landscape of the individual host response and outcomes in sepsis: a prospective cohort study. Lancet Respir. Med. 4, 259-271 (2016).

36. Burnham, K. L. et al. Shared and distinct aspects of the sepsis transcriptomic response to fecal peritonitis and pneumonia. Am. J. Respir. Crit. Care Med. 196, 328-339 (2017).

37. Scicluna, B. P. et al. Classification of patients with sepsis according to blood genomic endotype: a prospective cohort study. Lancet Respir. Med. 5, 816-826 (2017).

38. Sweeney, T. E. et al. Unsupervised analysis of transcriptomics in bacterial sepsis across multiple datasets reveals three robust clusters. Crit. Care Med. 46, 915-925 (2018).

39. Iglesias, J. et al. A 33-mRNA classifier is able to produce inflammopathic, adaptive, and coagulopathic endotypes with prognostic significance: the Outcomes of Metabolic Resuscitation Using Ascorbic Acid, Thiamine, and Glucocorticoids in the Early Treatment of Sepsis (ORANGES) Trial. J. Pers. Med. 11, 9 (2020).

40. Sweeney, T. E. et al. Validation of inflammopathic, adaptive, and coagulopathic sepsis endotypes in coronavirus disease 2019. Crit. Care Med. 49, e170-e178 (2021).

41. Wong, H. R. et al. Identification of pediatric septic shock subclasses based on genome-wide expression profiling. BMC Med. 7, 34 (2009).

42. Wong, H. R. et al. Validation of a gene expression-based subclassification strategy for pediatric septic shock. Crit. Care Med. 39, 2511-2517 (2011).

43. Wong, H. R. et al. Developing a clinically feasible personalized medicine approach to pediatric septic shock. Am. J. Respir. Crit. Care Med. 191, 309-315 (2015).

44. Wong, H. R. et al. Toward a clinically feasible gene expression-based subclassification strategy for septic shock: proof of concept. Crit. Care Med. 38, 1955-1961 (2010).

45. Wong, H. R. et al. Endotype transitions during the acute phase of pediatric septic shock reflect changing risk and treatment response. Crit. Care Med. 46, e242-e249 (2018).

46. Wong, H. R., Sweeney, T. E., Hart, K. W., Khatri, P. \& Lindsell, C. J. Pediatric sepsis endotypes among adults with sepsis. Crit. Care Med. 45, e1289-e1291 (2017).

47. Hotchkiss, R. S., Monneret, G. \& Payen, D. Sepsis-induced immunosuppression: from cellular dysfunctions to immunotherapy. Nat. Rev. Immunol. 13, 862-874 (2013).

48. Hutchins, N. A., Unsinger, J., Hotchkiss, R. S. \& Ayala, A. The new normal: immunomodulatory agents against sepsis immune suppression. Trends Mol. Med. 20, 224-233 (2014)

49. Venkatesh, B. \& Cohen, J. Hydrocortisone in vasodilatory shock. Crit. Care Clin. 35 263-275 (2019).

50. Annane, D. et al. Hydrocortisone plus fludrocortisone for adults with septic shock N. Engl. J. Med. 378, 809-818 (2018).

51. Annane, D. et al. Effect of treatment with low doses of hydrocortisone and fludrocortisone on mortality in patients with septic shock. JAMA 288, 862-871 (2002).

52. Sprung, C. L. et al. Hydrocortisone therapy for patients with septic shock. N. Engl. J. Med. 358, 111-124 (2008).

53. Venkatesh, B. et al. Adjunctive glucocorticoid therapy in patients with septic shock. N. Engl. J. Med. 378, 797-808 (2018).

54. Antcliffe, D. B. \& Gordon, A. C. Why understanding sepsis endotypes is important for steroid trials in septic shock. Crit. Care Med. 47, 1782-1784 (2019).

55. Gordon, A. C. et al. Effect of early vasopressin vs norepinephrine on kidney failure in patients with septic shock: the VANISH Randomized Clinical Trial. JAMA 316, 509-518 (2016). 
56. Antcliffe, D. B. et al. Transcriptomic signatures in sepsis and a differential response to steroids. From the VANISH Randomized Trial. Am. J. Respir. Crit. Care Med. 199, 980-986 (2019).

57. Wong, H. R., Hart, K. W., Lindsell, C. J. \& Sweeney, T. E. External corroboration that corticosteroids may be harmful to septic shock endotype a patients. Crit. Care Med. 49, e98-e101 (2021).

58. Wong, H. R. et al. Combining prognostic and predictive enrichment strategies to identify children with septic shock responsive to corticosteroids. Crit. Care Med. 44, e1000-e1003 (2016).

\section{ACKNOWLEDGEMENTS}

This work was funded by the National Institute of General Medical Sciences, R35GM126943 (to H.R.W.).

\section{COMPETING INTERESTS}

H.R.W. and the Cincinnati Children's Hospital Research Foundation hold US patents for the PERSEVERE biomarkers and the pediatric septic shock endotyping strategy described in the manuscript.

\section{ADDITIONAL INFORMATION}

Correspondence and requests for materials should be addressed to H.R.W.

Reprints and permission information is available at http://www.nature.com/ reprints

Publisher's note Springer Nature remains neutral with regard to jurisdictional claims in published maps and institutional affiliations. 